\title{
DESIGN SOCIAL: UM ESTUDO DE CASO DAS SALAS DE AULA DA FUNDAÇÃO CASA
}

Fabrício Mira

UNESP/FAAC - PPGDesign

fabriciomira@gmail.com

Ana Renata Mantova

UNESP/ FAAC - PPGDesign

anamantova@hotmail.com
Lílian Lago

UNESP/ FAAC - PPGDesign

lago.lilian@gmail.com

Rodrigo Gomes Curimbaba

UNESP/FAAC - PPGDesign

nunescurimbaba@hotmail.com

Prof. Dr. Olympio José Pinheiro

UNESP/FAAC - PPGDesign

holihn@uol.com.br

Resumo: A partir da segunda metade do século passado, o design passou a incluir no seu âmbito de atuação várias possibilidades de ação social. Muito mais do que gerar lucro para a indústria, o designer passa a atuar como um agente de transformação social, aplicando seus conhecimentos para minimizar problemas de grupos menos favorecidos. Com base nisso, este projeto tem como público-alvo adolescentes que se envolveram na criminalidade e hoje cumprem ações socioeducativas na Fundação CASA. O objetivo é investigar de que forma os estímulos visuais do ambiente das salas de aula atuam sobre os adolescentes e quais as possibilidades de intervenção, visando contribuir com o foco, a disposição e um melhor desempenho dos alunos durante atividades de aprendizagem. Tendo em vista a necessidade de construção de novas unidades da Fundação CASA, devido ao quadro de superlotação que o sistema apresenta atualmente, ressalta-se a importância de futuros estudos levarem estas reflexões à fase preliminar de projeto das futuras unidades.

Palavras-chave: design social, design de superfícies, percepção visual.

Abstract: From the second half of last century, the design has included in its scope of activity various possibilities of social action. Much more than generate profit for the industry, the designer starts to act as an agent of social transformation, applying their knowledge to minimize problems of disadvantaged groups. Based on this, this project has the target audience adolescents who engaged in crime and today meet social and educational activities in the CASA Foundation. The aim is to investigate how the environment of visual stimuli classrooms operate on teenagers and what the possibilities of intervention in order to contribute to the focus, layout and better performance of students during learning activities. In view of the need to build new units of the CASA Foundation, due to overcrowding above the system currently has, it emphasizes the importance of future 
studies bring these reflections to the project preliminary stage of these new units.

Palavras-chave: social design, surface design, visual perception.

\section{INTRODUÇÃO}

A partir da década de 70, o design passou a considerar várias possibilidades de atuação social, voltando sua atenção às necessidades de grupos sociais desfavorecidos, bem como à preservação da natureza. O design sustentável e humanitário, muito além de deixar-se conduzir por interesses econômicos do mercado, preocupa-se em gerar oportunidades de desenvolvimento, seguindo princípios de cidadania e ética (PAPANEK, 1977). De acordo com Pazmino (2007), o designer é um agente de transformação que atua de maneira ética, aplicando seus conhecimentos para minimizar problemas sociais por meio de técnicas de projeto adequadas a este objetivo.

$\mathrm{Na}$ década de 1930, durante a grande depressão econômica, o desenho industrial direcionou seus esforços para reduzir custos de fabricação, facilitar a utilização e a aparência dos produtos industrializados como forma de garantir uma maior possibilidade de vendas (PAZMINO, 2007). Mas após a retomada da economia, conforme relata Papanek (1977b), o design perdeu seu foco, ocupando-se em projetar trivialidades e extravagâncias.

Quando uma nova crise assolou os grandes centros econômicos na década de 1970, houve o surgimento de uma consciência social e ambiental por parte dos designers mais atuantes (HARVEY, 2007). Para o design, um dos marcos dessa década de recessão ocorreu na conferência realizada pelo Instituto Nacional de Design da Índia, em 1970. A Declaração de Amhedabad sobre o Design Industrial para o Desenvolvimento, assinada no evento, propõe que:

[...] o design poderia dar uma contribuição valiosa para o desenvolvimento econômico de uma nação e que a metodologia do design é inadequadamente conhecida e usada insuficientemente como um recurso econômico (DECLARAÇÃO DE AHMEDABAD apud MARGOLIN, 2009).

O trabalho do designer social envolve o contato contínuo com as comunidades mais desfavorecidas, por meio de um "trabalho de consultoria voluntária ou subsidiada por governos, ONGs ou empresas, nas mais variadas áreas de atuação" (PAZMINO, 2007). Ajudando indivíduos ou comunidades a resolverem seus problemas ou a criarem novas oportunidades, emerge o que Manzini (2008) define como inovação social. Dentre as possibilidades de ações orientadas à inovação social, encontram-se os projetos voltados para a inserção social, projetos voltados ao meio-ambiente (Figura 1), ações que alcancem repercussão social não intencional e projetos sociais intencionais (FORNASIER, MARTINS e MERINO, 2012). 


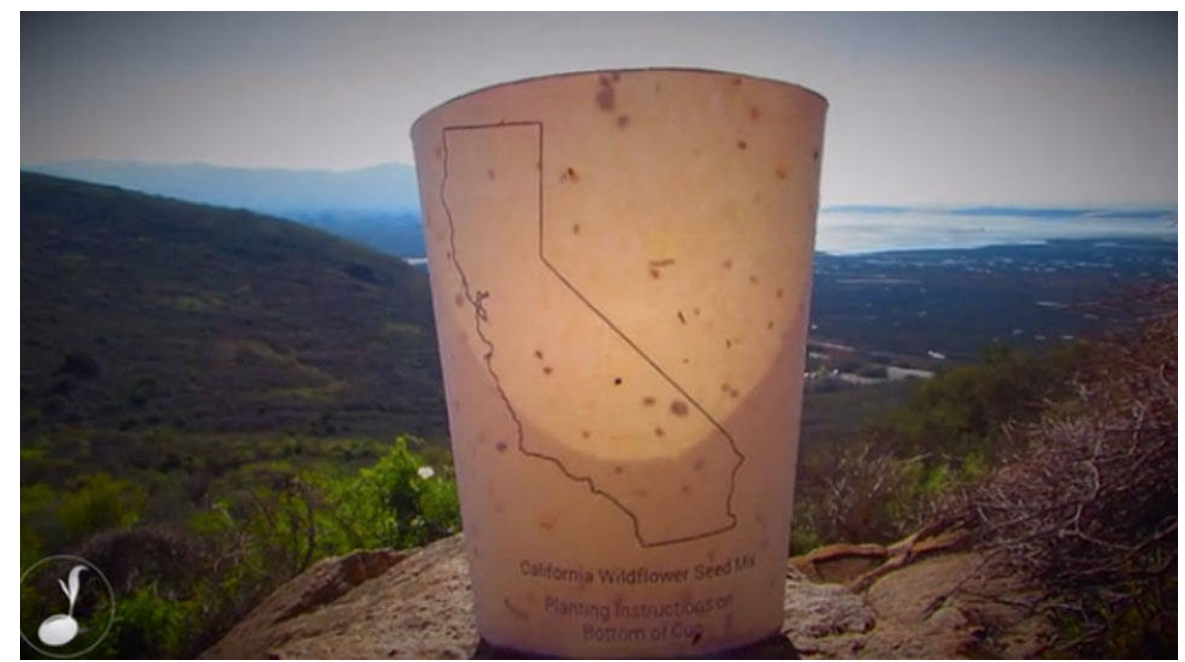

Figura 1 - A startup Reduce.Reuse.Grow. é um projeto de repercussão social voltado ao meioambiente. Em 2015, a empresa recebeu mais de $\mathbf{\$ 2 0 . 0 0 0 ~ e m ~ c o n t r i b u i c ̧ o ̃ e s ~ e s p o n t a ̂ n e a s ~ n o ~ s i t e ~ d e ~}$ crowdfunding kickstarter.com para a produção de copos de papel feitos com sementes que, quando descartados, germinam e geram árvores.

Fonte: REDUCE.REUSE.GROW (2016).

Para este estudo, destaca-se uma modalidade de inovação social baseada na manipulação de pessoas por meio subliminar voltada à inserção social do público-alvo. Esta categoria de projeto funda-se na premissa de que todo indivíduo está sujeito a estímulos sensoriais do ambiente e cabe ao designer intervir para otimizá-los, a fim de garantir o seu bem-estar.

Com base no escopo ideológico do design social, este estudo definiu como públicoalvo adolescentes de 12 a 21 anos que cumprem ações socioeducativas na Fundação CASA. As salas de aula, frequentadas diariamente por estes adolescentes, foram analisadas com o objetivo de investigar de que forma os estímulos visuais destes ambientes atuam sobre eles e quais as possibilidades de intervenção, visando a melhoria do foco, da disposição e do desempenho dos alunos durante atividades de aprendizagem.

\section{ESTUDO DE CASO - FUNDAÇÃO CASA}

A Fundação Centro de Atendimento Socioeducativo ao Adolescente (CASA), instituição vinculada à Secretaria de Estado da Justiça e da Defesa da Cidadania do Estado de São Paulo, foi criada com a missão de aplicar medidas socioeducativas, visando a capacitação e o retorno do adolescente à sociedade. A instituição está subordinada às diretrizes e normas previstas no Estatuto da Criança e do Adolescente e no Sistema Nacional de Atendimento Socioeducativo (SINASE). Até maio de 2015, foram implantados 72 pequenos centros socioeducativos em todo o Estado, principalmente no interior e no litoral. Destes, 61 unidades têm capacidade para atender até 56 adolescentes, sendo parte deles geridos em parceria com organizações da sociedade civil dos municípios onde foram implantadas (FUNDAÇÃO CASA, 2014).

Segundo Berenice Gianella (2011), presidente da Fundação CASA, os jovens da Fundação são filhos de famílias desestruturadas e sem acesso a serviços básicos, que abandonaram a escola sem que houvesse providências e que acabaram na criminalidade por não terem sido resgatados anteriormente. 
Marisa Fortunato (2011), superintendente pedagógica da Fundação, complementa:

Enquanto profissionais da educação, entendemos que há um ponto de partida, uma premissa já presente na Constituição de 1988 [...] da condição peculiar de pessoa em desenvolvimento. Assim, a legislação reconhece o papel imprescindível da educação para o desenvolvimento de crianças e adolescentes, em seu sentido mais amplo, prioritário e abrangente. Se, num passado recente, no Brasil a medida socioeducativa já teve natureza eminentemente repressora, hoje a mesma possui expressamente conteúdo ético-pedagógico assegurado pela legalidade.

De acordo com o Ministério Público de São Paulo, mais de $90 \%$ das casas de internação enfrentam um quadro de superlotação, e a construção de novas unidades se faz urgente. Para a demanda de 9.549 vagas, o sistema oferece apenas 8.079, gerando um déficit de 1.470 vagas, ou $18,19 \%$ do oferecido atualmente (TRUFFI, 2014).

A perspectiva de ampliação do sistema é uma oportunidade para que futuros estudos considerem a atuação de designers ainda na fase projetual, prioritariamente no projeto das salas de aula, uma vez que é nos ambientes de aprendizagem que ocorre o primordial papel da instituição: educar, capacitar e reinserir esses jovens na sociedade. Este estudo, porém, limita-se à análise e criação de uma proposta destinada às unidades já estabelecidas, tendo como modelo a unidade Morro Azul, em Limeira, a partir de fotos coletadas online.

\subsection{Análise das salas de aulas}

As Figuras 2 e 3 apresentam dois modelos de salas de aula da unidade Morro Azul, em Limeira. Constata-se a escassez - ou até mesmo a ausência - de uma fonte de luz natural no campo de visão dos alunos. Recentes estudos indicam que a iluminação do ambiente pode influenciar o comportamento de usuários e a sua disposição para atividades intelectuais. Baixo rendimento, inquietude e sensação de cansaço constantes podem ser componentes emocionais resultantes dos estímulos do ambiente (YAN et al., 2010; SHUAl, 2013).

Em 2011, Govén e seus colaboradores conduziram um estudo em uma escola secundária localizada no sul de Londres durante 6 meses, para testar a influência da iluminação do ambiente no desempenho de alunos em atividades acadêmicas como leitura, escrita e questões de matemática. Os resultados mostraram um progresso constante no desempenho dos alunos das salas onde houve uma intervenção para melhoria das condições de iluminação. Este estudo também revelou que a luz do dia parece influenciar os níveis de humor. Os níveis de cortisol foram maiores na classe com a melhoria da iluminação ambiente, ao longo dos 6 meses. Alterações na melatonina também foram constatadas. Diante disso, os pesquisadores indicam a necessidade de levar-se em conta os efeitos biológicos da iluminação do ambiente no projeto de ambientes de estudo para crianças. 


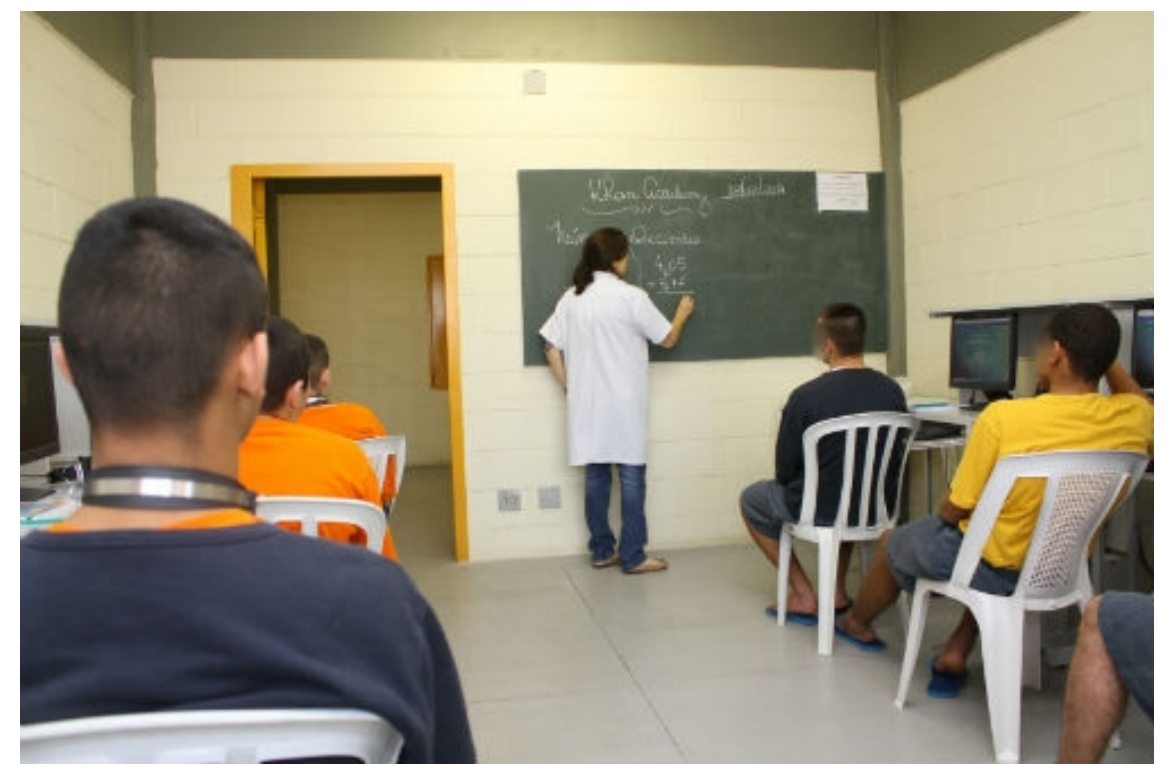

Figura 2 - Alunos do 9o ano do Ensino Fundamental cumprem medida socioeducativa na Fundação CASA, unidade Morro Azul em Limeira.

Fonte: PORTILHO, 2014.

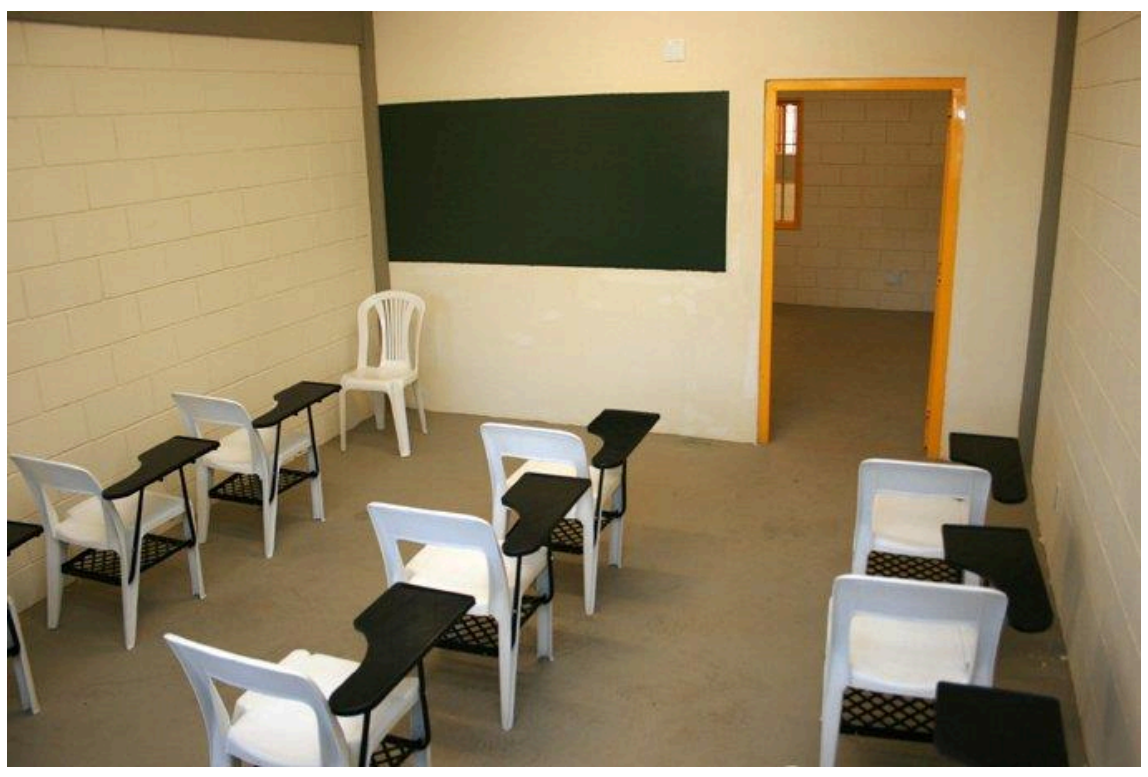

Figura 3 - Sala de aula da Fundação CASA, unidade Morro Azul em Limeira. Fonte: PORTILHO, 2014.

Além da iluminação ambiente, a visualização de imagens naturais pode ser um recurso efetivo na indução de reações psicológicas em usuários. Kaplan (1982) realizou uma série de experimentos sobre biofilia, com o objetivo de testar cientificamente essas reações. Esta hipótese foi originalmente proposta por Erich Fromm em seu livro The anatomy of human destructiveness, de 1973, mas ainda não havia sido testada empiricamente até 1982 . Os resultados revelam que ambientes ricos em imagens e paisagens naturais proporcionam benefícios emocionais aos usuários, reduzindo o estresse e aumentando a concentração. Lidwell, Holden e Butler (2010) sugerem que isso se deve porque o cérebro pode ter um processamento diferenciado para imagens naturais, como afirmam: 
A tendência inata a cenas verdes talvez tenha evoluído nos primeiros seres humanos porque conferia uma vantagem seletiva, um viés provavelmente relacionado à preferência pela savana.

Heerwagen e Hase (2001) listam outros resultados de pesquisas em biofilia:

- Janelas com vista para paisagens naturais reduzem o estresse no ambiente de trabalho.

- Árvores em locais de aglomerações humanas estão associadas ao aumento da interação social e do senso de comunidade em bairros urbanos.

- Observar peixes tropicais em um aquário reduz a pressão arterial.

- Pacientes em pós-operatório têm sua recuperação acelerada por visualizarem a natureza e a luz do dia.

Os autores destacam ainda que os benefícios ocorrem mesmo sem o uso de plantas reais no ambiente (Figura 4). Imagens projetadas, impressas ou em telas de computadores já seriam suficientes para induzir reações e são ideais para ambientes onde a aprendizagem, a reabilitação física, mental e a concentração sejam fundamentais (LIDWELL, HOLDEN, BUTLER, 2010).
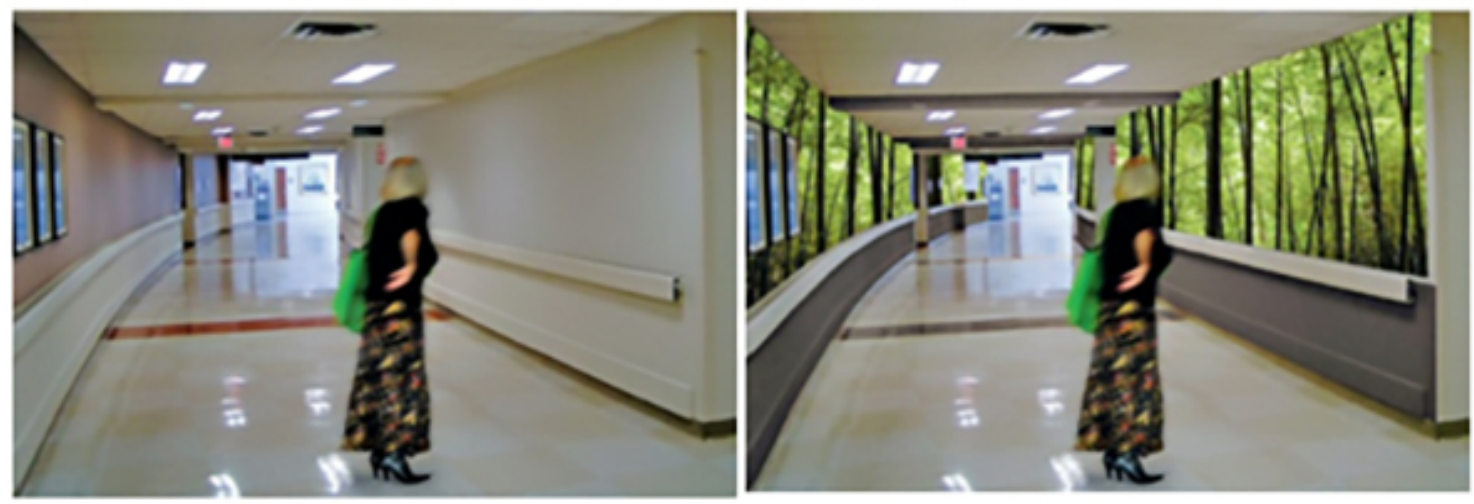

Figura 4 - Redesign do corredor de um hospital americano.

Fonte: LIDWELL, HOLDEN, BUTLER, 2010.

Estudos sobre o efeito das cores sobre a percepção dos usuários durante a execução de tarefas atestam os benefícios da biofilia. A cor pode agir como estímulo ambiental sutil e tem influências importantes sobre o comportamento.

Em testes realizados na Inglaterra com 20 estudantes, pesquisadores avaliaram se alterações na cor da iluminação do ambiente seriam capazes de influenciar o comportamento dos alunos. A coloração vermelha levou a um aumento dos níveis de distração dos estudantes. Mas a luz vermelha, quando combinada à luz incandescente, fluorescente e à luz do dia, produziu um aumento na produtividade (JOHNSON, 2013).

Elliot et al. (2007) cita quatro experimentos que demonstram que uma breve percepção do vermelho, por exemplo, prejudica a performance em testes de raciocínio - como o teste de QI - e este efeito parece atuar à revelia da consciência dos participantes. Estes achados sugerem que é preciso ter cuidado na forma como as cores são usadas em ambientes que exigem concentração na execução de tarefas.

Em outro estudo, 108 voluntários foram expostos a salas com paredes de cores diferentes. Com relação à sala com paredes verdes, as emoções mais relatadas foram 
de neutralidade e alegria, ao passo que e raiva, a surpresa, o medo e a tristeza foram as menos indicadas para esta cor.

\begin{tabular}{|l|c|c|c|c|c|c|c|}
\cline { 2 - 8 } \multicolumn{1}{|c|}{ Emotions } & \multicolumn{7}{l|}{} \\
\hline Living Rooms & Anger & Disgust & Neutral & Surprise & Happiness & Fear & Sadness \\
\hline RED room & & & & & & & \\
\hline GREEN room & & & & & & & \\
\hline BLUE room & & & $\square$ & & & & \\
\hline GRAY room & & & $\square$ & & & & \\
\hline
\end{tabular}

Figura 5 - Principais emoções associadas às salas com paredes de cores diferentes.

Fonte: HELVACIOĞLU, 2011.

\subsection{Redesign}

A partir das referências teóricas discutidas acima, foram definidas duas intervenções para as salas de aula analisadas. A primeira é a criação de uma fonte de luz no campo de visão periférica dos alunos, por meio da inserção de uma janela, cujo modelo leve em conta todos os requisitos de segurança exigidos. A segunda é a aplicação de imagens naturais com a predominância da cor verde na parede oposta à janela. Foi realizada uma pesquisa online para catalogar possíveis soluções para a intervenção na superfície da parede escolhida. Na Figura 6, essas imagens estão organizadas em uma sequência que se inicia com ilustrações vetoriais mais abstratas, seguindo gradualmente para imagens figurativas e realistas, até a representação fotográfica da natureza.

As ilustrações vetoriais abstratas e figurativas $a, b, c, d$, e e, foram descartadas por considerarmos que a representação realista preserva mais intensamente as propriedades biofílicas da imagem. Os módulos de repetição $f$ e $g$, embora realistas, geram padrões uniformes e monótonos, o que pode ocasionar no esgotamento do estímulo após um longo período de exposição. Segundo Berlyne (1971), imagens com um nível ótimo de complexidade são mais agradáveis e passíveis de serem contempladas por longos períodos.

A foto da imagem $h$ foi escolhida porque cumpre com os requisitos de complexidade, variedade, além de possuir uma carga simbólica relacionada à esperança e bem-estar. Além disso, a representação fotográfica impressa em alta definição evoca o sentido tátil, intensificando seus efeitos sobre os usuários. Pesquisas sobre a percepção sensorial frequentemente consideram que a visão e o tato trabalham com grande interdependência. O sentido do tato é mais intenso quando acompanhado pela visualização da imagem texturizada (ROBLES-DE-LA-TORRE, 2006). Em superfícies visuais-táteis, quando o estímulo visual é mais intenso e, portanto, mais confiável, o sentido tátil se adaptada para coincidir com a visão (BURGE et al., 2010). Este efeito pode se intensificar se a impressão gráfica for feita sobre um material com textura levemente granulada, onde imagem impressa seja complementada pelo sentido tátil. Há diversas opções no mercado de materiais autocolantes que simulam texturas de pedra, madeira e cimento, por exemplo. 


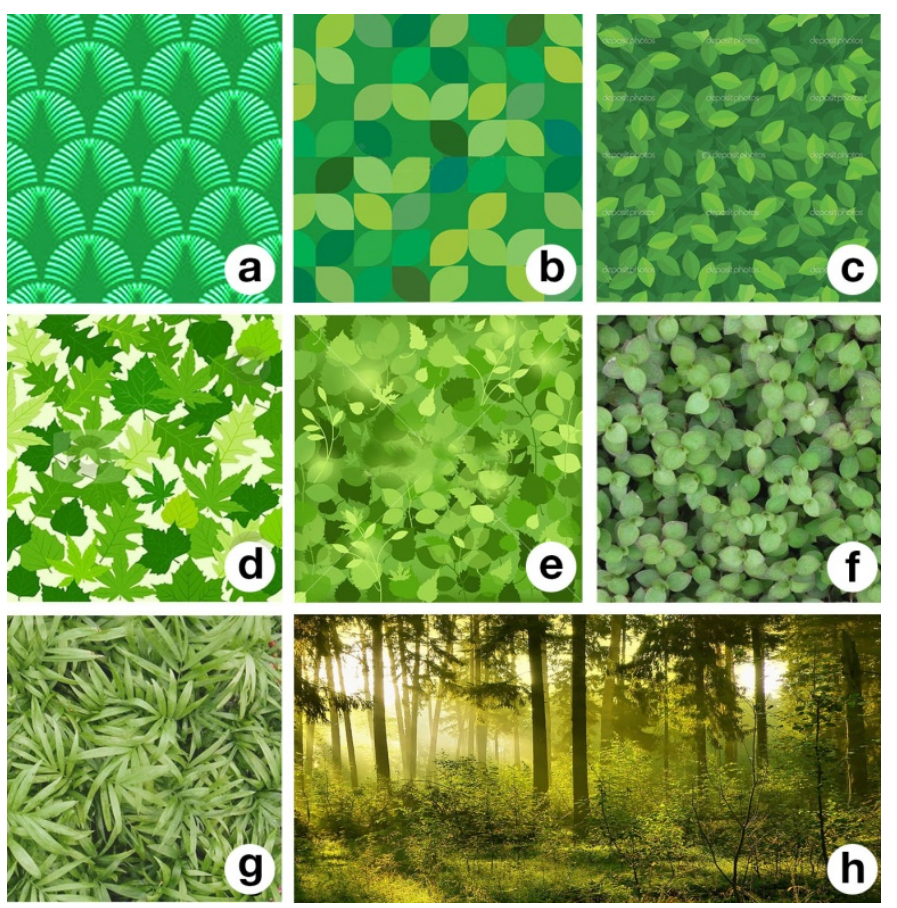

Figura 6 - De "a" a "g", módulos de repetição, rapport, ou seamless patterns, através dos quais é possível cobrir grandes superfícies com um mesmo padrão visual (RÜTHSCHILLING, 2008, p. 64). Fonte: http://br.depositphotos.com/

Recomenda-se que a imagem seja impressa em alta definição com tecnologia LATEX. Ao contrário das tintas à base de solventes, esta possui cores vivas e reais, é lavável, à prova de água e não tem cheiro, sendo ideal para o uso em ambientes internos. É também uma tecnologia de baixo custo, considerando que se trata de um projeto em grande escala.

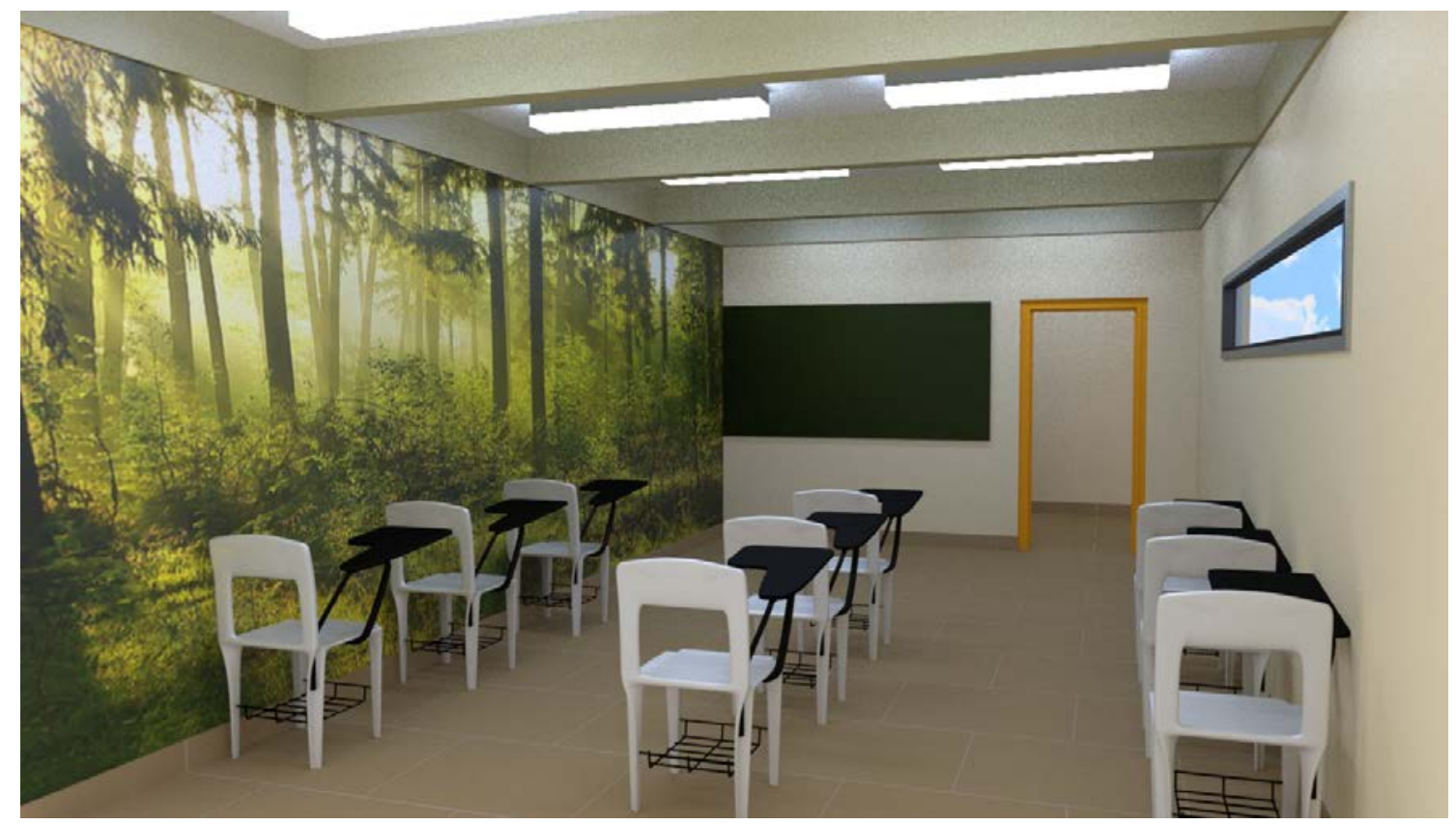

Figura 7 - Simulação digital da proposta de redesign da sala de aula da Fundação CASA de Limeira. Fonte: imagem elaborada pelos autores durante a pesquisa com base na Figura 3 (PORTILHO, 2014). 


\section{CONSIDERAÇÕES FINAIS}

Este estudo realizou uma análise de dois modelos de salas de aulas de uma unidade da Fundação CASA, com o objetivo de criar uma proposta de redesign que contribuísse com o foco, a disposição e o desempenho dos alunos, respeitando as limitações intrínsecas a uma instituição penitenciária pública, como segurança e baixo custo de implantação.

A solução apresentada baseia-se em estudos que atestam que ambientes com iluminação natural e a visualização de imagens da natureza promovem o bem-estar e a melhoria do desempenho de usuários na execução de tarefas de aprendizagem. 0 design, ao auxiliar na recuperação de jovens infratores, atua de forma direta na construção de uma sociedade mais justa e inclusiva.

Devido à superlotação constatada e diante da perspectiva de ampliação do sistema, surge uma oportunidade para que futuros estudos considerem a atuação de designers ainda na fase projetual, prioritariamente no projeto das salas de aula, uma vez que nestes ambientes concretiza-se o primordial papel da instituição: educar, capacitar e reinserir adolescentes na sociedade.

A revisão bibliográfica e a simulação digital aqui apresentadas não eliminam a necessidade da realização de testes controlados para avaliar empiricamente as soluções propostas.

\section{REFERÊNCIAS}

BERLYNE, Daniel E. Aesthetics and psychobiology. New York: Appleton-Century-Crofts, 1971.

BURGE, Johannes; GIRSHICK, Ahna R.; BANKS, Martin S. Visual-haptic adaptation is determined by relative reliability. The Journal of Neuroscience, v. 30, n. 22, p. 77147721, 2010.

ELLIOT, Andrew J. et al. Color and psychological functioning: the effect of red on performance attainment. Journal of experimental psychology: General, v. 136, n. 1, p. 154, 2007.

FORTUNATO, Marisa. Medidas socioeducativas e Educação: uma relação difícil mas possível. Fundação CASA, São Paulo, 04 jan. 2011. Disponível em: < http://www.casa.sp.gov.br/files/ Artigo_Marisa_Fortunato.pdf>. Acesso em 29 jul. 2015.

FUNDAÇÃO CASA - Centro de Atendimento Socioeducativo ao Adolescente, São Paulo, 23 mai. 2014. Disponível em: < http://www.fundacaocasa.sp.gov.br/View.aspx ?title=a-fun dação\&d=10>. Acesso em 29 jul. 2015.

GOVÉN, Tommy et al. Influence of ambient light on the performance, mood, endocrine systems and other factors of school children. CIE 27th Session, Sun City, South Africa, v. 112, 2011.

GIANELLA, Berenice. Acolher ou incriminar. Fundação CASA, São Paulo, 06 ago. 2011. Disponível em: <http://www.fundacaocasa.sp.gov.br/View.aspx?title=acolher-ou-incri minar\&d=900>. Acesso em 29 jul. 2015.

HELVACIOĞLU, Elif. Colour-emotion associations in interior spaces. Tese (doutorado). BILKENT UNIVERSITY, 2011. 
PORTILHO, Gabriela. Porque mais alunos da fundação casa estão gostando de aprender matemática. Instituto NET, Claro e Embratel, 22 set. 2014. Disponível em: <https://www.institutoclaro.org.br/reportagens-especiais/por-que-mais-alunos-dafun dacao-casa-estao-gostando-de-aprender-matematica/>. Acesso em: 29 jul. 2015.

JOHNSON, Cynthia S.; RUITER, Gÿsbert A. Envisioning Classroom Design with light and colour. Academic Research International, v. 4, n. 4, p. 550, 2013.

MANZINI, Ezio. A matéria da invenção. Lisboa: Centro Português de Design, 1993.

OZTÜRK, E. The Effects of Color Scheme on the Appraisal of an Office Environment and Task Performance. 2010. Tese de Doutorado. Master Thesis Supervised by Yilmazer S, Bilkent University, Ankara.

PAPANEK, Victor. Como cometer um crimen: las responsabilidades sociales y morales del diseñador. In: Deseñar para El Mundo Real: ecologia humana y cambio social. Madrid: Hermann Blume, 1977.

REDUCE.REUSE.GROW. Compostable Plantable Coffee Cups. Disponível em: <http://www.planttrash.com/>. Acesso em: 24 fev. 2016.

ROBLES-DE-LA-TORRE, Gabriel. The importance of the sense of touch in virtual and real environments. leee Multimedia, n. 3, p. 24-30, 2006.

VALDEZ, Patricia; MEHRABIAN, Albert. Effects of color on emotions. Journal of Experimental Psychology: General, v. 123, n. 4, p. 394, 1994.

RÜTHSCHILLING, Evelise Anicet. Design de superfície. Porto Alegre: Ed. da UFRGS, 2008.

SHUAI, Gao. A Research Review of Classroom light environment. China Illuminating Engineering Journal, p. S1, 2013.

TRUFFI, Renan. MP denuncia governo Alckmin e Fundação CASA e fala em "nova Febem". Carta Capital. São Paulo: Editora Confiança Ltda, 2014. Disponível em: http://www.cartacapital.com.br/revista/812/ministerio-publico-denuncia-governoalckmin-e-fundacao-casa-e-fala-em-nova-febem-9382.html

YAN, Yong-hong et al. Productivity and Physiological Response of Students Subjected to Fluorescent Lamps With Different Colour Temperatures and Luminance Level [J]. Journal of Civil, Architectural \& Environmental Engineering, v. 4, p. 017, 2010. 